\title{
Observation of the Cosmic-Ray Shadow of the Moon and Sun with IceCube
}

\author{
F. Bos ${ }^{1, a}$, F. Tenholt ${ }^{1, a}$, J. Becker Tjus ${ }^{1, a}$, and S. Westerhoff ${ }^{2,3, a}$ \\ ${ }^{1}$ Department of Physics, Ruhr-Universität Bochum, 44801 Bochum, Germany \\ ${ }^{2}$ Department of Physics, University of Wisconsin, Madison, WI 53706, USA \\ ${ }^{3}$ Wisconsin IceCube Particle Astrophysics Center, Madison, WI 53703, USA \\ ${ }^{a}$ For the IceCube Collaboration, http://icecube.wisc.edu
}

Correspondence to: F. Bos (fabian.bos@icecube.wisc.edu)

Received: 27 April 2015 - Revised: 18 June 2015 - Accepted: 25 June 2015 - Published: 5 August 2015

\begin{abstract}
Moon shadow analyses are standard methods to calibrate cosmic-ray detectors. We report on a threeyear observation of cosmic-ray Moon and Sun shadows in different detector configurations. The cosmic-ray Moon shadow was observed with high statistical significance $(>6 \sigma)$ in previous analyses when the IceCube detector operated in a smaller configuration before it was completed in December 2010. This work shows first analyses of the cosmic-ray Sun shadow in IceCube. A binned analysis in one-dimension is used to measure the Moon and Sun shadow with high statistical significance greater than $12 \sigma$.
\end{abstract}

\section{Introduction}

IceCube is a Cherenkov light detector with an instrumented volume of $1 \mathrm{~km}^{3}$ at the geographic South Pole. The main goal is to detect high energy neutrinos. The trigger rate is dominated by muons produced by cosmic-rays interacting with the Earth's atmosphere. A deficit of cosmic-rays from the direction of the Moon and Sun is expected and was observed for the Moon in Aartsen et al. (2014) with greater than $6 \sigma$.

Other experiments used the Moon shadow to calibrate their detectors as well (di Sciascio and Iuppa, 2011; Karelin et al., 2015). The Sun has not been used as a calibrator of the IceCube detector before. Its shadow is expected to be influenced by the solar magnetic field. A Sun shadow analysis was performed for the Tibet AS-Gamma experiment (Amenomori et al., 2013). The influence of the solar magnetic field was clearly visible, seen in two-dimensional maps presented in Amenomori et al. (2013). The depth of the cosmic-ray Sun shadow decreases from the year 1996 $(\approx 6 \%)$ to $2001(\approx 2 \%)$ and increases then to $2009(\approx 6 \%)$ (Amenomori et al., 2013). An anticorrelation between the number of Sun spots and the depth of the Sun shadow was also seen in the Tibet AS-Gamma experiment (Amenomori et al., 2013). The cosmic-ray shadow of the Moon, on the other hand, is observed as almost steady with slight uncer- tainties. The effect for the Sun is expected to be caused by the deflection of charged cosmic-rays in the magnetic field close to the solar surface.

Simulations near the source surface for different models (CSSS and PFSS) are compared with measured data from the Tibet AS-Gamma experiment in Amenomori et al. (2013).

The IceCube Moon and Sun shadow analysis is based on a binned analysis which compares on- and off-source regions to compute relative deficit of events at the expected position of the celestial body.

\section{Detector configuration and data sample}

\subsection{IceCube detector}

The IceCube detector is located at the geographic South Pole, containing 86 strings deployed in glacial ice. High-energy neutrinos interact with protons and produce leptons that emit Cherenkov light. Digital Optical Modules (DOMs) deployed into the ice then detect these photons.

The entire detector consists of 4800 DOMs in 80 strings with a $125 \mathrm{~m}$ horizontal spacing and $17 \mathrm{~m}$ vertical spacing between strings (Abbasi et al., 2010). A more compact group of strings that contains 360 additional DOMs is located at the bottom of the detector and is called DeepCore (Abbasi et al., 
2012). Altogether, IceCube is operating with 86 strings containing 5160 DOMs. Before IceCube was completed in December 2010, it was operating in smaller detector configurations.

\subsection{Data sample}

For the detector configuration with 40 and 59 strings (IC40 and IC59) a Moon shadow analysis was done in Aartsen et al. (2014). This work makes use of the data taken from detector configurations with 79 and 86 strings (IC79 and IC86-I). Moreover, the second year (IC86-II) in which the detector was operating in its final configurations has been analyzed. A data selection for the IC40 and IC59 detector configuration is described in Aartsen et al. (2014). At the South Pole Moon and Sun filters are enabled if the celestial body is at least $15^{\circ}$ above the horizon (Aartsen et al., 2014). The Moon reaches this threshold for at least 6 days each month.

The Sun, on the other hand, reaches this threshold for three consecutive months during the Antarctic summer, from mid November though mid February each year. To reconstruct the trajectory of a charged particle travelling through the detector, the Multi-Photo-Electron (MPE) fit is used. The MPE fit takes into account all photons that reach a DOM.

Compared with the Single Photo-Electron (SPE) fit, which only uses the arrival time of the first photon in each DOM, the MPE fit yields a slightly better angular resolution in highenergy range, indicated by simulation studies (Aartsen et al., 2014).

\section{Simulation}

Simulation studies are necessary to improve the quality of the significance of the Moon and Sun shadow analyses. CORSIKA is used to simulate cosmic-rays and their decays on their way to the IceCube detector. Muons are produced in interactions of comic-rays with particles in the Earth's atmosphere.

In Aartsen et al. (2014), simulation studies estimate a median energy for primary particles of $20 \mathrm{TeV}$ that trigger the IceCube detector. However, the mean energy for primary particles that also pass the Moon and Sun filters is $40 \mathrm{TeV}$ with $68 \%$ between 10 and $200 \mathrm{TeV}$ (Aartsen et al., 2014).

An energy range of $600 \mathrm{GeV}$ to $100 \mathrm{TeV}$ in simulation studies is used to optimize quality cuts.

\subsection{Quality cuts}

Reconstruction quality cuts optimize the statistical significance because events with high uncertainty in the reconstruction are excluded in this analysis where two cut variables are used: The angular uncertainty in the muon reconstruction $\left(\sigma_{i}\right)$ and the reduced $\log$-likelihood $(\operatorname{rog} \mathrm{L})$.
Both variables are used for standard selection cuts in neutrino analyses (Abbasi et al., 2011). Poisson statistics are assumed resulting in a proportionality of the statistical significance $S$ to the fraction of events passing the cuts $\eta$ and the resulting median angular resolution $\Psi_{\text {med }}$ after cuts:

$S \propto \frac{\sqrt{\eta}}{\Psi_{\text {med }}}$.

In both detector configurations (IC79 and IC86) the quality cuts are $\sigma<0.71^{\circ}$ and $\operatorname{rog} \mathrm{L}<8.1$.

\section{Binned analysis}

A binned analysis is used to measure the shadowing effect of the Moon and Sun.

\subsection{Description of the method}

The 1-D analysis compares one on-source region and eight off-source regions. The on-source region is a window of $\pm 5^{\circ}$ around the expected position of the Moon and Sun. With an offset of $\pm 5^{\circ}, \pm 10^{\circ}, \pm 15^{\circ}$ and $\pm 20^{\circ}$ the off-source regions are defined. In each bin $(i)$ the relative deficit is computed by:

$\frac{\Delta N_{i}}{\langle N\rangle}=\frac{N_{i}^{\text {on }}-\left\langle N_{i}^{\text {off }}\right\rangle}{\left\langle N_{i}^{\text {off }}\right\rangle}$,

with $N^{\text {on/off }}$ as all events in on-source/off-source regions. The uncertainty is given by

$\sigma_{\Delta N /\langle N\rangle}=\frac{N_{i}^{\text {on }}}{\left\langle N_{i}^{\text {off }}\right\rangle} \sqrt{\frac{1}{N_{i}^{\text {on }}+\frac{1}{8 \cdot\left\langle N_{i}^{\text {off }}\right\rangle}} .}$

In Aartsen et al. (2014), the Moon is described as a pointsource sink that reduces the muon sample by $\Phi \pi R_{\mathrm{M}}^{2}$ events. The same approach can be used in this analysis, the radius of the Moon and Sun is given given by $R_{\mathrm{M} / \mathrm{S}} \approx 0.26^{\circ}$.

Following Aartsen et al. (2014) further, the point spread function (PSF) can be described by a two-dimensional Gaussian. Assuming that the PSF is azimuthally symmetric, the relative deficit can be fit with a Gaussian function that depends only on the radial distance $\Psi$ (Aartsen et al., 2014),

$f(\Psi)=-\frac{\Phi \pi R_{\mathrm{M} / \mathrm{S}}^{2}}{\sigma^{2}} \cdot e^{-\Psi^{2} / 2 \sigma^{2}}$.

Thus, the relative deficit can be described as (Aartsen et al., 2014)

$\Delta N=-\frac{R_{\mathrm{M} / \mathrm{S}}^{2}}{2 \sigma^{2}} e^{-\Psi_{i}^{2} / 2 \sigma^{2}}$. 

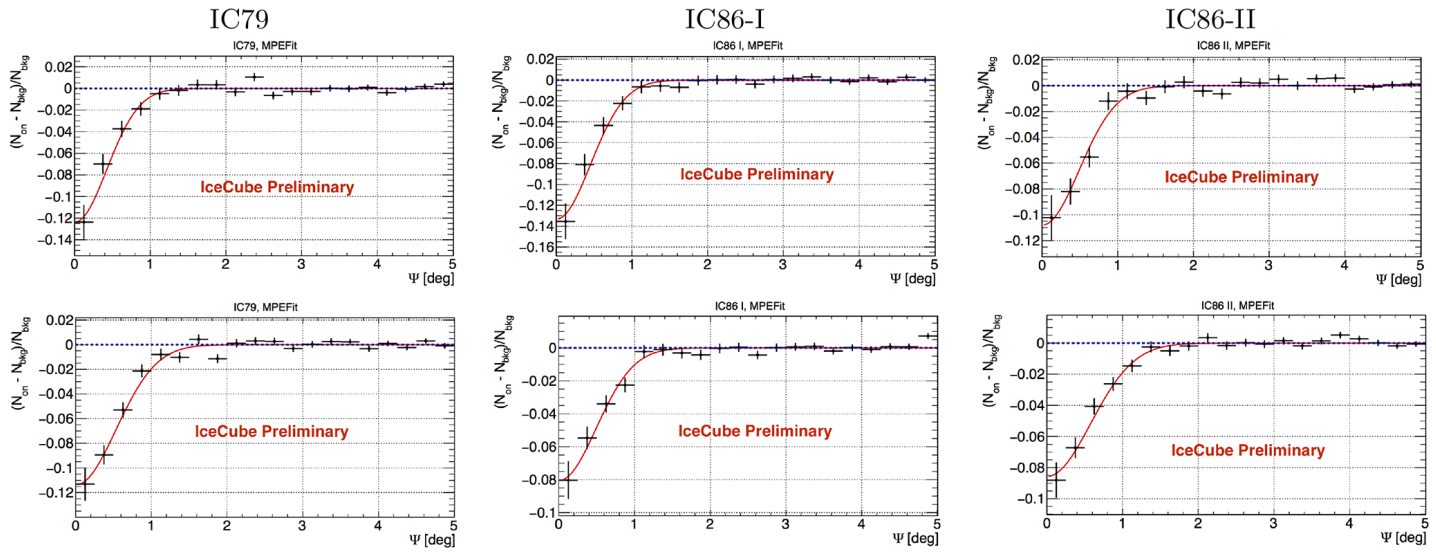

Figure 1. Relative deficit in the number of events in on-source and off-source regions as a function of the radius $(\Psi)$. First row shows Moon shadow, second row Sun shadow.

Table 1. Results of the one-dimensional analysis, with $A$ as the amplitude and $\sigma$ as the width of the Gaussian.

\begin{tabular}{lllcccc}
\hline \multirow{2}{*}{ Year } & $\sigma_{\text {gauss }} \Delta\left(\sigma_{\text {gauss }}\right)$ & $A$ & $\Delta(A)$ & $S$ \\
\cline { 3 - 5 } & \multicolumn{3}{c}{$\left({ }^{\circ}\right)$} & & & \\
\hline \multirow{3}{*}{ Moon } & IC79 & 0.43 & 0.05 & 0.12 & 0.02 & $>12 \sigma$ \\
& IC86-I & 0.45 & 0.05 & 0.13 & 0.03 & $>13 \sigma$ \\
& IC86-II & 0.49 & 0.05 & 0.11 & 0.02 & $>12 \sigma$ \\
\hline \multirow{3}{*}{ Sun } & IC79 & 0.53 & 0.05 & 0.11 & 0.01 & $>16 \sigma$ \\
& IC86-I & 0.49 & 0.06 & 0.08 & 0.01 & $>13 \sigma$ \\
& IC86-II & 0.57 & 0.05 & 0.09 & 0.01 & $>16 \sigma$ \\
\hline
\end{tabular}

\subsection{Results}

Approximately $30 \%$ of all events pass the quality cuts each year and are included in Moon and Sun shadow analyses. Results can be found in Table 1 and Fig. 1 .

The statistical significance $S$ is computed by the difference between the $\chi_{\text {Line }}^{2}$ of a line and the $\chi_{\text {gaussian }}^{2}$ of a Gaussian that are fitted to data:

$\frac{\Delta \chi^{2}}{\Delta \text { dof }}=\frac{\chi_{\text {gaussian }}^{2}-\chi_{\text {Line }}^{2}}{\text { dof }}$.

Here, dof are the degrees of freedom. The difference $\Delta$ dof is given by 1 . Thus, the $p$ value is computed via $\Delta \chi^{2}$ and results in the significance $S$ :

$S=\sqrt{2} \cdot \operatorname{erf}^{-1}(1-p)$.

Here, $\operatorname{erf}^{-1}$ is the Inverse Error Function and $p$ the $p$ value.

A smaller time binning of these results can be found in Table 2. The results show a constant angular resolution $\sigma$ with error bars for each celestial body.

Both celestial bodies are observed with high statistical significance $(>12 \sigma)$. These significances are higher for both detector configurations (IC79 and IC86) as compared to
Table 2. Results of the one-dimensional analysis in a smaller time binning. $A$ is the amplitude of the fitted Gaussian.

\begin{tabular}{lcc}
\hline Month & Rel. deficit $A$ & $\Delta A$ \\
\cline { 2 - 3 } & \multicolumn{2}{c}{$(\%)$} \\
\hline November 2010 & 13.3 & 3.2 \\
December 2010 & 9.2 & 1.4 \\
January 2011 & 15.1 & 2.5 \\
November 2011 & 8.3 & 2.3 \\
December 2011 & 9.1 & 1.6 \\
January 2012 & 6.5 & 1.7 \\
November 2012 & 9.3 & 2.1 \\
December 2012 & 7.1 & 1.1 \\
January 2013 & 9.0 & 1.7 \\
\hline
\end{tabular}

IC40. A statistical significance of more than $12 \sigma$ was also observed in IC59 (Aartsen et al., 2014).

The Moon shadow shows a steady amplitude of the fitted Gaussian where all values are within the statistical uncertainties and there is no statistically significant variation of the depth of the solar shadow at this point.

However, detailed information is not possible at this point and more studies are necessary to indicate a possible influence of the solar magnetic field. Additionally, there is a big difference between the Tibet AS-Gamma Experiment and Sun shadow analysis of the IceCube detector. The Tibet ASGamma Experiment could observe the Sun shadow the entire year but IceCube's observation time is shorter with approximately 90 days during the antarctic summer. Figure 2 shows the predicted and measured number of Sun spots by NASA and the median times corresponding to IceCube Sun shadow observations with different detector configurations.

Comparing the relative deficit maps in Amenomori et al. (2013) and Fig. 2, the anticorrelation between the number of Sun spots and the amplitude of the cosmic-ray Sun shadow 


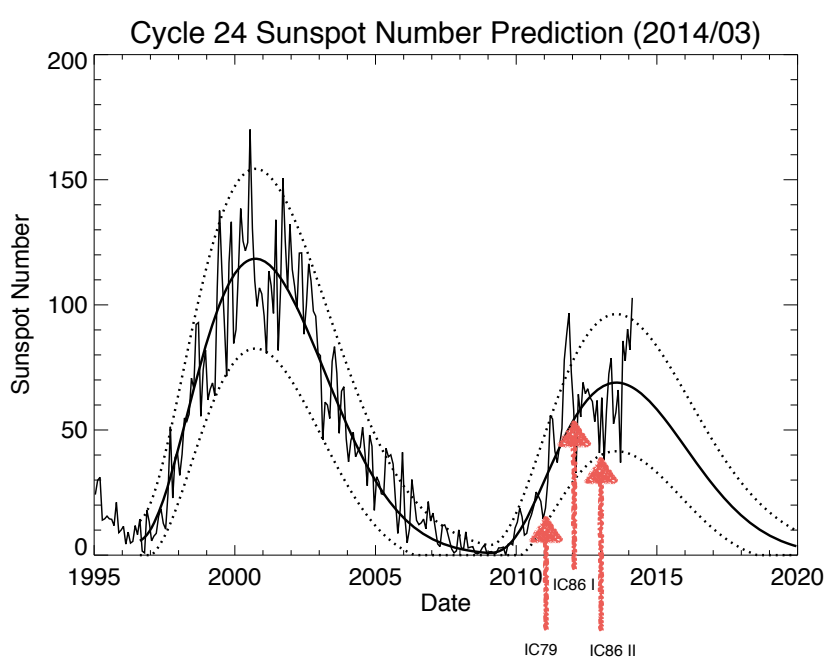

Figure 2. Figure shows predicted and measured Sun spot cycle by NASA. Red Arrows illustrate the IceCube observation times for the cosmic-ray Sun shadow. Figure modified from http://solarscience. msfc.nasa.gov.

is clearly visible. The Tibet AS-Gamma experiment observed the Sun shadow with a maximum of 170 Sun spots in the year 2000.

IceCube's Sun shadow observation period is during relatively lower Sun activity as seen in Fig. 2, where a maximum of 100 Sun spots is seen in IC86-I (2012). Furthermore, Fig. 2 illustrates that the number of Sun spots vary in a short time frame.

\section{Conclusion and outlook}

\subsection{Conclusion}

The shadowing effect of the Moon and Sun of cosmic-rays in the $\mathrm{TeV}$ range is observed with high statistical significance $(>12 \sigma)$. Data were taken from IC79 and two years of the final configuration IC86, during periods between June 2010 and May 2013 in which these celestial bodies were well above the horizon. Under the assumption that the sample of the IC40 and IC59 configuration are similar, the angular resolution is slightly improved.

In Aartsen et al. (2014), an angular resolution of (0.71 \pm $0.07)^{\circ}$ and $(0.63 \pm 0.04)^{\circ}$ are observed. For the Moon in IC79 and IC86 an average angular resolution of $(0.46 \pm 0.05)^{\circ}$ is measured. Additionally, the angular resolution of the three year period is constant within the statistical uncertainties.

The depth of the shadow is illustrated as the amplitude of the fitted Gaussian, which could be of big interest in future studies. An almost stable amplitude of the Moon shadow of $0.12 \pm 0.02$ is shown in Table 1 .

\subsection{Outlook}

Further studies of upcoming years (IC86-III and IC86-IV) are necessary to investigate an influence of the solar magnetic field. In particular, it will be necessary to analyse each month separately in order to investigate the correlation between the magnetic field strength during that period and the depth of the shadow. In Amenomori et al. (2013), the Tibet ASGamma experiment simulated particles influenced by different source surface (SS) models with different radii. For IceCube, it is also necessary to compute the expected Moon and Sun shadow within these models. Two-dimensional analyses are useful to make the shadows visible. A binned smoothing method and an unbinned analysis are options for this purpose.

An unbinned analysis was performed in Aartsen et al. (2014) for the IC40 and IC59 configuration.

Acknowledgements. We acknowledge the support from BMBF, 05A14PC1, and Marcos Santander from Columbia University, New York.

Edited by: K. Scherer

Reviewed by: K. Alexander and one anonymous referee

\section{References}

Aartsen, M. G., Abbasi, R., Abdou, Y., et al. (IceCube Collaboration): Observation of the cosmic-ray shadow of the Moon with IceCube, Phys. Rev. D, 89, 102004, doi:10.1103/PhysRevD.89.102004, 2014.

Abbasi, R., Abdou, Y., Abu-Zayyad, T., et al. (IceCube Coll.): Calibration and Characterization of the IceCube Photomultiplier Tube, Nucl. Instrum. Meth. A,, 618, 139-152, doi:10.1016/j.nima.2010.03.102, 2010.

Abbasi, R., Abdou, Y., Abu-Zayyad, T., et al. (IceCube Collaboration): Time-integrated Searches for Point-like Sources of Neutrinos with the 40-string IceCube Detector, Astrophys. J., 732, 18, doi:10.1088/0004-637X/732/1/18, 2011.

Abbasi, R., Abdou, Y., Abu-Zayyad, T., et al.: The design and performance of IceCube DeepCore, Astropart. Phys., 35, 615-624, doi:10.1016/j.astropartphys.2012.01.004, 2012.

Amenomori, M., Bi, X. J., Chen, D., et al.: Probe of the Solar Magnetic Field Using the "Cosmic-Ray Shadow" of the Sun, Phys. Rev. Lett., 111, 011101, doi:10.1103/PhysRevLett.111.011101, 2013.

di Sciascio, G. and Iuppa, R.: Observation of the Cosmic Ray Moon shadowing effect with the ARGO-YBJ experiment, Contribution to the 32nd International Cosmic Ray Conference, Beijing, 1118 August 2011, 1-34, doi:10.7529/ICRC2011/V01/0226, 2011.

Karelin, A. V., Voronov, S. A., and Koldobskiy, S. A.: Observation of the Moon's shadow in cosmic rays with energies above $400 \mathrm{GeV}$ in the PAMELA satellite experiment, Cosmic Res., 53, 128-132, doi:10.1134/S0010952515020045, 2015. 